Working Paper

Ambivalent Reactions to People Who Deny or Admit Their Gender and Racial Biases

\author{
*Sylvia Perry ${ }^{1}$ \\ Allison L. Skinner ${ }^{1,3}$ \\ James E. Wages ${ }^{1}$ \\ Johannes Parzonka ${ }^{2}$ \\ ${ }^{1}$ Psychology Department \\ Northwestern University \\ 2029 Sheridan Rd. \\ Evanston, IL 60208 \\ ${ }^{2}$ Psychology Department \\ Leiden University, \\ Wassenaarseweg 52 \\ 2333 AK Leiden \\ The Netherlands \\ ${ }^{3}$ Psychology Department \\ University of Georgia \\ 125 Baldwin Street \\ Athens, GA 30602
}

*Corresponding author: sylvia.perry@northwestern.edu, 847-467-6454 


\begin{abstract}
In the face of evidence that someone is socially biased or prejudiced, we examined how people react to those who deny or admit having such flaws. We proposed the novel hypothesis that people's perceptions and evaluations of individuals who deny or admit their social biases may be ambivalent. As such, we hypothesized that when a denier and an admitter of social bias would be considered together, people would react to the denier more negatively than to the admitter. However, when a denier and an admitter would be considered independently, we predicted the individual's self-report about whether or not they were biased would be accommodated, such that the denier would be perceived as less prejudiced than the admitter. In eight studies $(N=$ 3,933), we tested this hypothesis for both gender and racial biases, across participant gender and race, and with multiple indicators of bias (i.e., an implicit bias test; subtle and blatant hiring discrimination). Findings across studies provided robust support for our hypothesis, suggesting that people have mixed reactions to individuals who deny or admit their social biases.
\end{abstract}

KEYWORDS: Prejudice, bias, perceptions 


\section{Ambivalent Reactions to People Who Deny or Admit Their Gender and Racial Biases}

Imagine an individual on a hiring committee who is presented with evidence suggesting that they made a biased judgement when evaluating job applicants, unfairly preferring applicants of one social group (e.g., men or White people) over another (e.g., women or Black people). This individual might respond to this feedback by denying that they have any biases or acknowledging their biased behavior. How do others perceive an individual who denies or admits that they might be biased? On the one hand, others might value the fact that an individual is willing to admit their faults and also praise their self-awareness and motivation to improve. However, it is uncommon for people to admit that they are socially biased, and when people do admit that they hold such biases, they may suffer negative consequences such as being labeled as prejudiced or bigoted. Thus, on the other hand, some might perceive the individual who denies, compared to admits, their bias in a more positive light, assuming that they must be less prejudiced. Another possible outcome is that people may be ambivalent about how they perceive the individual who admits their bias, recognizing that a person who is willing to admit their faults is perhaps more trustworthy and honest (John, Barasz, \& Norton, 2016), while at the same time critically judging them based on their biases. These perceptions may vary depending on the contexts in which the judgments are made. Here we examine whether - in the face of evidence that someone has engaged in sexist or racist behavior-people evaluate the individual who denies, compared to admits, their bias more positively, and the circumstances under which this may occur.

To the extent that acknowledgement of having social biases is perceived as a step toward prejudice reduction (Moskowitz et al., 2011; Monteith \& Mark, 2009), those who deny having biases may be perceived as more prejudiced than those who admit. In general, people tend to 
evaluate those who admit their mistakes and other vulnerabilities fairly positively (Bruk, Scholl, \& Bless, 2018), value others who are willing to be honest about their negative attributes, and trust people who reveal their negative attributes more than those who conceal their negative attributes (John, Barasz, \& Norton, 2016; Wheeless \& Grotz, 1977). For example, when there is evidence of an individual's unintentional wrongdoing, they are perceived to have more integrity when they admit, rather than deny, their mistakes (Kim, Ferrin, Cooper, \& Dirks, 2004). Moreover, admitting the existence of one's biases is often assumed to be the first step to reducing them, which is a common-sense notion reflected in most strategies of organizational diversity trainings (Noon, 2018). These findings suggest that people would appreciate others' willingness to admit their social biases, and prefer them to those who deny and conceal their biased tendencies.

Despite the value placed on admitting one's own negative attributes and mistakes, admission of faults that are considered deeply undesirable may not be so advantageous. The expression of overt social bias has become increasingly unpopular over the last several decades (Dovidio \& Gaertner, 2000; Spence \& Hahn, 1997; Twenge, 1997). Calling someone prejudiced is considered highly offensive, and potentially a more offensive social violation than sexist and racist behaviors or statements, themselves (Augoustinos \& Every, 2010; Blaut, 1992). This may explain why it is not uncommon to hear people make disclaimers like "I'm not racist, but..." before going on to say something many other people would consider racially biased (Augoustinos \& Every, 2010; Bonilla-Silva, 2002). As blatant expressions of sexism and racism are considered strongly taboo in contemporary American society, admitting engagement in prejudicial behavior likely carries severe social ramifications that ultimately outweigh the value 
of being forthright with one's faults. Therefore, people may have strong motivations to deny having such biases.

If an individual denies being socially biased, then observers may view them as less prejudiced than those who admit having such biases. For example, people are prone to believe what other people say about themselves (Bem, 1972), and often take it at face value, unless they are given reason to believe otherwise (Gilbert \& Malone, 1995). Moreover, the evaluability hypothesis suggests that, when information about an individual is limited and there is greater ambiguity, people may tend to overweight the information that is more accessible and more evaluable (Hse, 1996). Additionally, self-perception theory suggests that outside observers tend to think that individuals have better insight into their own internal states than outside observers do, particularly when the information about internal states are ambiguous (Bem, 1972). Thus, in the case of third-party bias attribution, there may be social motivation to trust others' personal assessments of their own biases. That is, because the social cost of attributing bias to others is so high, people may be particularly inclined to form or temper their judgments based on the individuals' own expressed self-perceptions and awareness (Redford \& Ratliff, 2016).

But might there be circumstances under which people's concerns about attributing bias to the denier are reduced? Work in economics and organizational behavior provides some insight into contexts in which people might evaluate the person who admits, relative to denies, their bias more positively. Specifically, there is a large body of work showing that people tend to evaluate information differently in contexts in which decisions are made jointly, versus separately (Bazerman et al., 1999). For example, when people are making separate evaluations, they tend to weight what they want to do over what they should do. In contrast, when making joint decisions, people may value what they should do over what they want to do. In this case, we would expect 
that, because avoiding being associated with a prejudiced individual is self-serving, participants might want to avoid and negatively evaluate the individual who admits their bias when making separate judgments, while recognizing that they should punish the person who denies their bias in situations in which there is a joint evaluation. Moreover, when people are making joint evaluations, they can weigh both pieces of information against one another and make a relative judgment, but when they are making separate evaluations, they might weight their perception of that information against a norm (Kahneman \& Miller, 1986). In this case, we would expect that people would more negatively evaluate the admitter relative to the denier in a separate decision because it is not normative for people to express that they are racially biased (Bonilla-Silva, 2002). In contrast, when these individuals are rated jointly and the biased behavior is held constant, the positive attribute of admitting fault might become more heavily weighted, and thus people may more positively evaluate the person who admits their prejudice.

With the current work we propose the novel hypothesis that, denying (compared to admitting) one's biases may have mixed consequences, in that, although people may favor those who admit biases to those who deny them, they may also tend to give those who deny their bias the benefit of the doubt. Specifically, we posit that these evaluations may be differentially weighted depending on whether the admitter and the denier are evaluated jointly versus separately. We expect that (H1), when two individuals (a denier and an admitter) are evaluated at the same time (and thus perceived prejudice is held constant), the value of admitting one's faults may become more salient, such that people will evaluate the individual who admits that they are biased more positively. However, we also hypothesize that $(\mathrm{H} 2)$ when people are evaluating a single individual who has engaged in biased behavior, they will shift the standard for prejudice depending upon the individual's self-report about whether or not they are biased. That is, we 
expect that in contexts in which participants either evaluate the admitter or the denier, they will give individuals who deny their biases the benefit of the doubt and thus perceive them to be less prejudiced, on average, and in turn more good and less worthy of social distancing than those who admit their biases. To date, no research of which we are aware has tested these questions.

In eight studies $(N=3,933)$, we tested these hypotheses. First, we investigated whether, when people are asked to compare two individuals — one who denied and one who admitted their bias (in the face of evidence that they are biased) - they would view the individual who denied their bias more negatively than the individual who admitted their bias (Studies 1a and 1b). Next, we examined judgments of one individual who either denied or admitted their bias (in spite of evidence that they were biased or engaged in hiring discrimination), testing (a) whether participants perceived the individual who denies being biased as less prejudiced, and (b) whether perceived prejudice predicted judgments of how good a person the individual was and the extent to which they were someone to socially avoid (Studies 2a-3d).

We tested these questions with multiple forms of evidence that an individual held biases (psychological test results, subtle hiring discrimination, and blatant hiring discrimination) and examined whether this occurred when the target of bias was a member of the participants' outgroup or a member of the participant's ingroup. In all studies, participants accessed the study online and provided informed consent before beginning the study; the university's Institutional Review Board approved all materials and procedures.

In Study 1, we sought to assess whether participants would evaluate the admitter more positively than the denier when making a relative comparison between two individuals who both were said to have shown biased behavior (such that biased behavior is held constant). Specifically, we assessed whether participants would indicate that they (Study 1a) and others 
(Study 1b) would perceive the admitter as less prejudiced, more good, and less worthy of social distancing than the denier. We also assessed whether this would vary as a function of the strength of the evidence of bias provided (blatant or subtle discrimination).

Participants learned that we were interested in how they (or other people) might judge people who made evaluations in a hiring scenario. They then read about the hiring scenario and learned that an evaluator supposedly read over 20 applications for a leadership position in an organization and recommended 10 of those applicants for employment. All participants learned that the pool had an equal number of Black people and White people, then they were randomly assigned to read that the evaluator had either (a) engaged in a subtle form of discrimination in which the Black and White applicants were equally qualified but the evaluator selected eight White people and only two Black people or (b) a more blatant form of discrimination in which the Black applicants were objectively more qualified but that the evaluator selected all 10 White people and no Black people. Next, participants were told that the evaluator was confronted with the biases in their decisions and had responded in one of two ways to this feedback: admitted to having a racial bias (indicating that they were working on it) or denied having a racial bias. Participants were then asked whether the admitter or denier was more prejudiced, a better person, and who they would most want to distance themselves from. For each of these outcomes, participants could select one of three relative responses: the admitter was more [prejudiced] than the denier, both the admitter and denier were equally [prejudiced], or the denier was more [prejudiced] than the admitter. A series of chi-square tests of goodness of fit were performed to determine whether the three relative responses were equally preferred for each of the primary outcomes of interest. 
For perceptions of prejudice, we collapsed across both conditions of blatant and subtle instances as responses did not significantly differ by discrimination level, $X^{2}(2, N=400)=1.52$, $p=.468$. Preference for the three relative responses was not equally distributed in the sample. As such, only $12.3 \%$ of participants viewed the bias admitter as more prejudiced than the bias denier; whereas, $47.8 \%$ of participants viewed the bias denier as more prejudiced than the bias admitter, $X^{2}(2, N=400)=83.62, p<.001$. In support of our first hypothesis, the admitter was perceived as less prejudiced than the denier when making relative comparisons.

Because evaluative outcomes tended to vary by the discrimination level for good person judgments $\left(X^{2}(2, N=401)=8.08, p=.018\right)$ and for social distancing $\left(X^{2}(2, N=401)=5.22, p\right.$ $=.074)$, we report the results of both outcomes separately for the blatant and subtle bias conditions. In the blatant discrimination condition, most participants $(65.7 \%)$ tended to think that the admitter was a better person than the denier, with only $5.5 \%$ of participants judging the bias denier as a better person than the bias admitter, $X^{2}(2, N=201)=111.08, p<.001$. Few participants (only 10.9\%) indicated more desire to socially distance themselves from the bias admitter relative to the bias denier; whereas, $49.3 \%$ of participants indicated more desired to socially distanced themselves from the bias denier relative to the bias admitter, $X^{2}(2, N=201)=$ 48.03, $p<.001$. In the subtle discrimination condition, the majority of participants $(52.5 \%)$ thought that the admitter was a better person than the denier, only $5.1 \%$ of participants thought that the bias denier was a better person than the bias admitter, $X^{2}(2, N=198)=74.30, p<.001$. Few participants (13.1\%) indicated more desire to socially distance themselves from the bias admitter relative to the bias denier; whereas, $37.9 \%$ of participants indicated more desire to socially distance themselves from the bias denier than the bias admitter, $X^{2}(2, N=198)=40.03$, $p<.001$. See Figure 1 for a graphical representation of findings. 
Given that perceptions of how others would respond to the evaluator did not vary by discrimination level for prejudice perceptions $\left(X^{2}(2, N=401)=0.59, p=.745\right)$, for good person judgments $\left(X^{2}(2, N=401)=1.03, p=.597\right)$, and for social distancing $\left(X^{2}(2, N=401)=2.99, p\right.$ $=.225$ ), we report the results of all outcomes collapsed across both blatant and subtle bias conditions. Only $14.7 \%$ of participants thought that others would perceive the bias admitter as more prejudiced than the bias denier; whereas, $50.1 \%$ of participants thought that others would perceive the denier as more prejudiced than the admitter, $X^{2}(2, N=401)=76.03, p<.001$. Most participants $(61.1 \%)$ also tended to think that others would perceive the blatant bias admitter to be a better person than the blatant bias denier, with only $9.5 \%$ of participants believing that others would perceive the denier as a better person than the admitter, $X^{2}(2, N=401)=163.04, p$ $<.001$. Likewise, few participants $(16.5 \%)$ predicted that others would desire more social distance from the blatant bias admitter than the blatant bias denier; whereas, $51.9 \%$ of participants predicted that others would desire more social distanced from the denier than the admitter, $X^{2}(2, N=401)=75.93, p<.001$. 

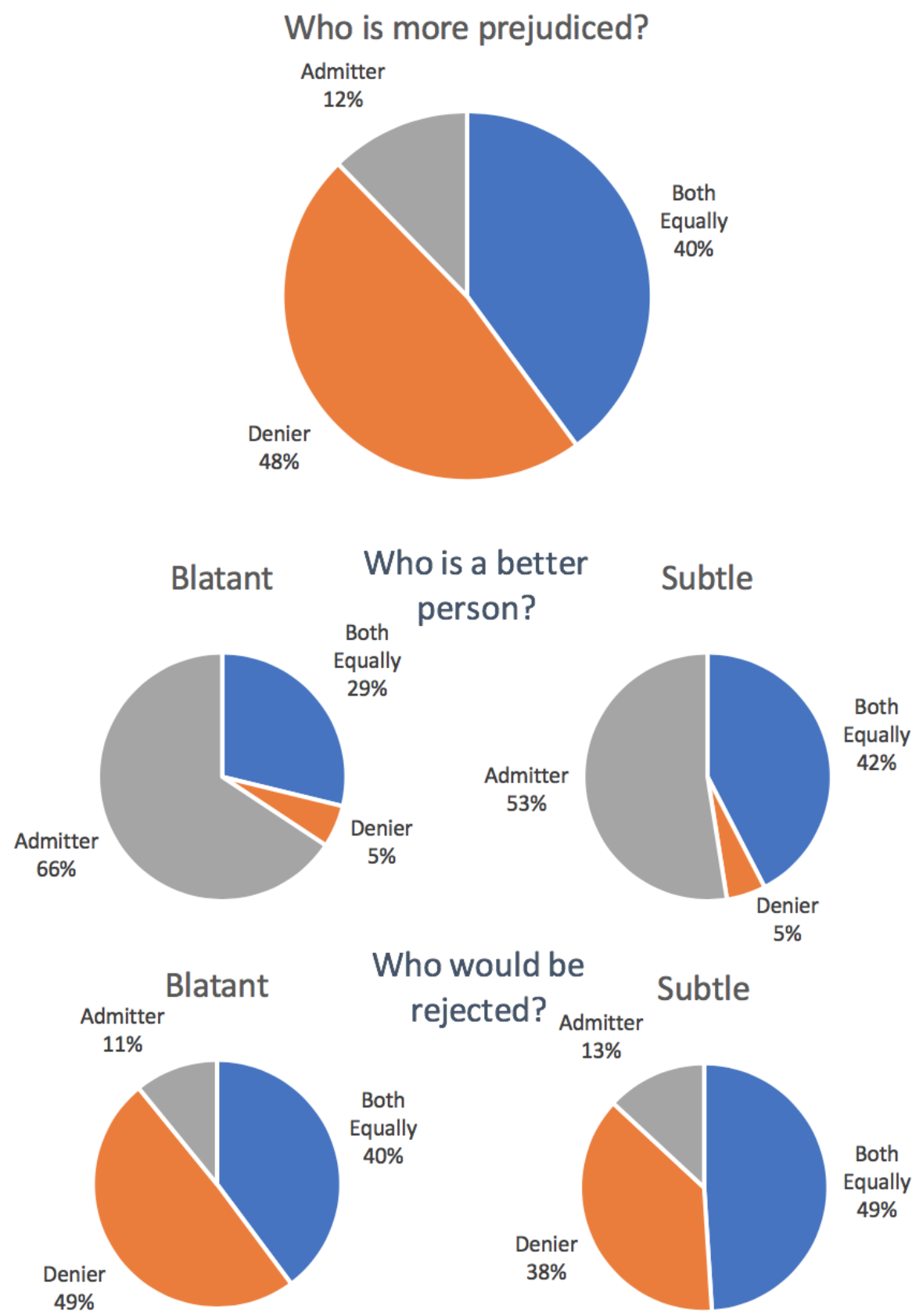

Fig. 1. Results of Study 1a. Charts present the distribution of participants that selected the denier (orange), admitter (grey), or both equally (blue) in response to who is more prejudiced, a better person, and social distanced at blatant and subtle levels of discrimination.

Study 1 established that, when making relative comparisons between someone who admits having biases and someone who denies having biases (after engaging in biased behavior), 
people favor, and also anticipate that others will favor, the admitter. Moreover, this holds regardless of whether the biased behavior was subtle or blatant. With Studies 2 and 3, we sought to examine the factors that might emphasize the negative aspects of admitting bias. We expected that when people evaluated a single individual who either admitted or denied their bias, they would be more likely to take the individual's response at face value, perceiving the individual who admitted bias to be more prejudiced. We investigated a range of biases based on both gender and race, with multiple forms of evidence of bias (psychological test results, subtle hiring biases, and blatant hiring biases). Moreover, we examined whether attitudes toward those who admitted versus denied their biases varied as a function of whether the bias was against the participants' own group or another group. We hypothesized that participants who read about an individual who denied having biases (in spite of evidence of their biases) would perceive the individual as less prejudiced than participants who read about an individual who admitted their biases, and that this would lead the individual who denied having biases to be evaluated as a better person, and prompt participants to desire less social distance from that individual.

In Studies $2 \mathrm{a}$ and $2 \mathrm{~b}$, we examined how participants perceived individuals who denied (vs. admitted) bias based on evidence from a psychological test (a measure of implicit social cognition). Participants were told that they had been assigned to learn information about someone who scored as having (a) "a strong automatic preference for White people over Black people" (Study 2a) or "a strong automatic preference for men over women in positions of leadership" (Study 2b) and (b) relatively low self-esteem. Moreover, to examine whether denying or admitting bias drove effects (if they were present) we included a number of control conditions. In Study 2a we include two control conditions; the individual showing evidence of bias never explicitly admitted or denied having biases in either control condition, but in one 
condition they admitted to having low self-esteem (another negative trait) and in the other they admitted nothing. In Study $2 \mathrm{~b}$ we eliminated the condition in which the individual stated nothing, but varied whether the individual indicated that they were working on the bias that they admitted or did not mention anything about working on the bias that they admitted. In all studies participants were asked to rate the individual that they were assigned to evaluate on their level of prejudice, how good of a person they are, and how much they would like to socially distance from that individual. We hypothesized that the individual who denied having (gender or racial) bias would be perceived as less prejudiced than individuals in the other conditions, and that this would lead the individual who denied having biases to be evaluated as a better person, and prompt participants to desire less social distance from that individual. Given the hypothesized indirect effects through perceived prejudice, we report the results of perceptions of prejudice and mediation models in the main text, all other analyses are reported in the supplemental file.

In Study 2a, a one-way analysis of variance (ANOVA) with response condition as the between-subjects factor revealed a significant main effect, such that perceptions of how prejudiced the individual is against Black people significantly varied as a function of response condition, $F(3,156)=14.67, p<.001, R^{2}=.22$. Bonferroni corrected pairwise comparisons indicated that the individual who denied that they were biased was perceived to be less prejudiced $(M=4.11, S D=1.24)$ than the individual who admitted that they were biased $(M=$ $5.54, S D=1.27 ; p<.001,95 \%$ CI $[0.75,2.11])$, the individual who admitted nothing $(M=5.59$, $S D=0.97 ; p<.001,95 \%$ CI $[0.80,2.15])$, and the individual who admitted low self-esteem $(M=$ $4.93, S D=0.96 ; p=.009,95 \%$ CI $[0.14,1.50])$.

Next, we tested for evidence that perceptions that the individual is prejudiced against Black people leads to participants' (a) perceptions that the individual is a good person and (b) 
social distancing, using statistical mediation. For the mediation models, we specifically focused on the two response conditions of greatest theoretical interest- (a) the person who admitted their biases and was working on mitigating their bias and (b) the person who denied having biases. Mediation analyses comparing evaluations of individuals who denied having bias to those who (a) admitted low self-esteem and (b) admitted nothing can be found in the supplement (for this and all other studies). We conducted mediation analyses using the PROCESS MACRO for SPSS 2.16.3 (Hayes, 2017).

Results from 5000 Bootstrap samples (bias corrected) indicated that the direct effect of response condition on perception that the individual is a good person was not significant, $B=$ $-0.03, S E=0.22, p=.907,95 \%$ CI $[-0.46,0.40]$. The confidence interval for the indirect effect did not contain 0 , indicating a significant indirect effect on perceptions that the individual is a good person through perceptions that the individual is prejudiced, $B=-0.30, S E=0.12,95 \% \mathrm{CI}$ $[-0.60,-0.10]$. The direct effect of response condition on social distancing was not significant, $B$ $=-0.21, S E=0.24, p=.400,95 \% \mathrm{CI}[-0.69,0.28]$. The confidence interval for the indirect effect did not contain 0 , indicating there was also a significant indirect effect on social distancing through perceptions that the individual is prejudiced, $B=0.49, S E=0.17,95 \% \mathrm{CI}[0.23,0.90]$.

In Study 2b, a one-way ANOVA with response condition as the between-subjects factor revealed a significant main effect, such that perceptions that the individual is prejudiced against women significantly varied as a function of response condition, $F(3,320)=16.25, p<.001, R^{2}=$ .13. Bonferroni corrected pairwise comparisons indicated that the individual who denied that they were biased was perceived to be significantly less prejudiced $(M=4.39, S D=1.26)$ than the person who admitted that they were biased and were working on it $(M=5.49, S D=1.06 ; p<$ $.001,95 \% \mathrm{CI}[0.59,1.62])$ and the person who admitted that they were biased but did not mention 
working on it $(M=5.47, S D=1.10 ; p<.001,95 \%$ CI $[0.57,1.59])$. Participant gender did not moderate these effects, $F(3,316)=2.23, p=.085, R^{2}=.16$.

Next, we tested whether perceptions that the individual is prejudiced against women mediated participants' (a) perceptions that the individual is a good person and (b) social distancing. Consistent with Study 2a, we tested the same conceptual model, investigating participants' evaluations of how good of a person the individual was and desire for social distance from deniers relative to admitters who stated that they were working on reducing their biases. Results from 5000 Bootstrap samples (bias corrected) indicated a significant direct effect of response condition on perception that the individual is a good person, $B=0.47, S E=0.16, p=$ $.004,95 \%$ CI $[0.16,0.79]$. However, the confidence interval for the indirect effect did not contain 0 , indicating a significant indirect effect on perceptions that the individual is a good person through perceptions that they are prejudiced, $B=-0.22, S E=0.07,95 \%$ CI $[-0.38$, $-0.11]$. The direct effect of response condition on social distancing was not significant, $B=$ $-0.20, S E=0.17, p=.242,95 \%$ CI $[-0.53,0.13]$. The confidence interval for the indirect effect did not contain 0 , indicating a significant indirect effect on social distancing through perceptions that the individual is prejudiced, $B=0.30, S E=0.08,95 \% \mathrm{CI}[0.16,0.48]$.

Study 2 provided evidence that in isolation (when evaluating a single individual) denying that one has biases reduces perceived prejudice, and mediation analyses supported the notion that this reduced social distancing and increased perceptions that the individual is a good person. In Study 3 we examined whether participants would show the same type of reaction to behavioral manifestations of bias. Specifically, participants read about a person who discriminated against job applicants who were applying for a position of leadership. In Study 3a, an equal number of male and female participants evaluated a scenario in which women (relative to men) had been 
discriminated against; in Study $3 \mathrm{~b}$ a predominantly White sample of participants evaluated a scenario in which Black (relative to White) applicants had been discriminated against. Finally, in Studies 3c and 3d, samples of Black and White participants (respectively) evaluated the same hiring scenario in which members of their own racial ingroup had been discriminated against.

In Study 3a, participants were informed that they had been assigned to read personal information about someone who had previously read over 20 applications for a leadership position in an organization and selected ten of those applicants. Participants learned that the pool had an equal number of men and women, and that the applicants were equally qualified. Further, participants were told the individual had selected eight men and only two women for leadership positions, suggesting that they are biased and have a preference for men over women in positions of leadership. Then, participants were randomly assigned to view one of three responses that the individual had to that information, the individual was said to have: (a) admitted having bias and indicated that they were working on it, (b) admitted having bias (but did not mention working on it), or (c) denied having bias.

In Study 3a, a one-way ANOVA with response condition as the between-subjects factor revealed a significant main effect, such that perceptions that the individual is prejudiced against women applicants significantly varied as a function of response condition, $F(2,240)=36.62, p<$ $.001, R^{2}=.22$. Bonferroni corrected pairwise comparisons indicated that the individual who denied that they were biased was perceived to be significantly less prejudiced $(M=4.35, S D=$ 1.38) than the person who admitted that they were biased and were working on it $(M=5.46, S D$ $=1.19 ; p<.001,95 \%$ CI $[0.66,1.55])$ and the individual who admitted that they were biased but did not mention working on it $(M=5.83, S D=0.88 ; p<.001,95 \%$ CI $[1.03,1.92])$. 
Next, we tested whether perceptions that the individual is prejudiced against women applicants mediated participants' (a) perceptions that the individual is a good person and (b) social distancing. Consistent with Studies $2 \mathrm{a}$ and $2 \mathrm{~b}$, here we focus on the individual who denied having biases relative to the individual who admitted their biases. Results from 5000 Bootstrap samples (bias corrected) indicated that the direct effect of response condition on perception that the individual is a good person was non-significant, $B=0.28, S E=0.17, p=.100,95 \% \mathrm{CI}$ $[-0.05,0.62]$. The confidence interval for the indirect effect did not contain 0 , indicating a significant indirect effect through perceptions that the individual is biased, $B=-0.34, S E=0.09$, $95 \%$ CI $[-0.54,-0.19]$. In addition, the direct effect of response condition on social distancing was not significant, $B=-0.30, S E=0.17, p=.079,95 \% \mathrm{CI}[-0.64,0.04]$. The confidence interval for the indirect effect did not contain 0 , indicating a significant indirect effect through perceptions that the individual is biased, $B=0.34, S E=0.10,95 \% \mathrm{CI}[0.16,0.58]$.

With Study 3b, we sought to investigate whether the findings from Study $3 a$ would replicate when Black, relative to White, people were discriminated against. We also added a blatant bias condition to Study 3b, to assess whether the effects would replicate when the individual engaged in a more blatant form of discrimination. The method for Study $3 \mathrm{~b}$ was identical to that of Study 3a, except that we dropped the condition in which the individual admitted bias but did not mention working on it, and participants were randomly assigned to read about subtle (as presented in Study 3a) or blatant discrimination. In the blatant bias condition participants were told that the ten Black people were objectively more qualified for the position than the ten White people (although the individual evaluating them was not explicitly informed of that) and that the individual evaluating them recommended hiring ten White people and zero Black people for the leadership job. 
In Study 3b, a $2 \times 2$ ANOVA with response condition (deny bias; admit bias but working on it) and discrimination level (subtle, blatant) as between-subjects factors revealed main effects of response condition, $F(1,803)=140.28, p<.001, \eta p 2=.149$, and discrimination level, $F(1$, $803)=51.30, p<.001, \eta p 2=.060$. These main effects were qualified by an interaction between response condition and discrimination level, $F(1,803)=10.37, p=.001, \eta p 2=.013$.

To decompose the interaction, a series of Bonferroni corrected pairwise comparisons were performed. In the subtle discrimination condition, the bias denier $(M=4.33, S D=1.63)$ was perceived to be significantly less prejudiced than the bias admitter $(M=5.72, S D=1.09 ; p<$ $.001,95 \%$ CI $[-1.65,-1.13])$. In the blatant discrimination condition, the bias denier $(M=5.29$, $S D=1.44)$ was also perceived to be significantly less prejudiced than the bias admitter $(M=$ $6.08, S D=0.96 ; p<.001,95 \% \mathrm{CI}[-1.05,-0.54])$. We also noted that, despite evidence of blatant discrimination, the bias denier was still perceived as significantly less prejudiced than the bias admitter who engaged in subtle discrimination $\left(M_{\text {diff }}=-0.43, S E=0.13, p=.006,95 \%\right.$ CI $[-0.78$, -0.09]; see Figure 1). 

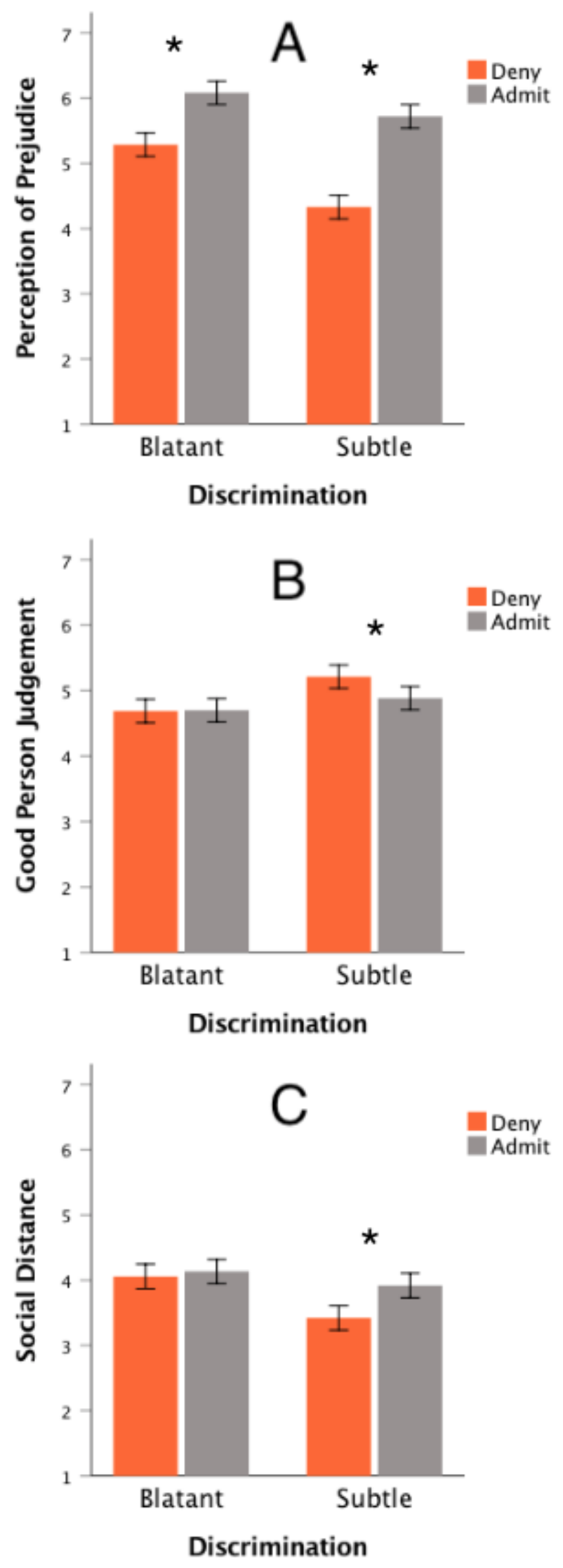
Fig. 1. Results of Study 3b. Graphs present judgments of the bias denier (orange) and admitter (grey) who engaged in different levels of discrimination (blatant, subtle). Bars represent perceptions of the evaluator as prejudiced (A), judgements of the evaluator as a good person (B), and desire for social distance away from the evaluator $(\mathbf{C})$. Error bars represent the upper and lower boundaries of the $95 \%$ confidence intervals. Asterisks indicate statistical significance below the .05 level.

Next, we tested whether perceptions that the individual is prejudiced against Black people mediated participants' (a) perceptions that the individual is a good person and (b) social distancing. Further, we tested whether this mediation was moderated by discrimination level (subtle, blatant). Using model 7 (moderated mediation) of the PROCESS procedure, results from 5000 Bootstrap samples (bias corrected) indicated that the direct effect of response condition on perception that the individual is a good person was significant, $B=0.23, S E=0.09, p=.011$, $95 \%$ CI $[0.05,0.42]$. The confidence interval for indirect effects in both the blatant $(B=-0.29$, $S E=0.05,95 \% \mathrm{CI}[-0.39,-0.19])$ and the subtle $(B=-0.50, S E=0.08,95 \%$ CI $[-0.66,-0.36])$ discrimination conditions did not contain 0 , indicating significant indirect effects through perceptions that the individual is prejudiced. The indirect effect was significantly stronger in the subtle discrimination condition than the blatant discrimination condition $\left(B_{\text {diff }}=-0.22, S E=0.07\right.$, $95 \%$ CI $[-0.37,-0.08])$. The direct effect of response condition on desire to socially distance from the individual was also significant, $B=-0.26, S E=0.09, p=.005,95 \%$ CI $[-0.43,-0.08]$. The confidence interval for indirect effects in both the blatant $(B=0.39, S E=0.07,95 \% \mathrm{CI}$ $[0.27,0.53])$ and the subtle $(B=0.69, S E=0.09,95 \% \mathrm{CI}[0.53,0.87])$ discrimination conditions did not contain 0 , indicating significant indirect effects through perceptions that the individual is 
prejudiced. The indirect effect was significantly stronger in the subtle discrimination condition than the blatant discrimination condition $\left(B_{\text {diff }}=0.30, S E=0.10,95 \%\right.$ CI $\left.[0.19,0.49]\right)$.

With Study 3c, we sought to investigate whether the findings from Study $3 b$ would replicate among predominantly Black participants. We tested whether participants would privilege the individual who denies having biases over the bias admitter, even when presented with evidence of discrimination toward the participant's racial ingroup. The method for Study $3 \mathrm{c}$ was identical to that of Study 3b, except that Black participants represented the vast majority of the sample $(\sim 99 \%)$.

In Study $3 \mathrm{c}$, a $2 \times 2$ ANOVA with response condition and discrimination level as between-subjects factors revealed main effects of response condition, $F(1,795)=70.25, p<$ $.001, \eta p 2=.081$, and discrimination level, $F(1,795)=24.89, p<.001, \eta p 2=.030$. These main effects were not qualified by a significant interaction between response condition and discrimination level, $F(1,795)=3.20, p=.074, \eta p 2=.004$. As such, the bias denier $(M=5.27$, $S D=1.52)$ was perceived as less prejudiced against Black people than the bias admitter $(M=$ $6.09, S D=1.27)$. Further, the subtle discriminator $(M=5.44, S D=1.46)$ was perceived as less prejudiced than the blatant discriminator $(M=5.93, S D=1.42)$.

Next, we tested whether perceptions that the individual is prejudiced against Black people mediated participants' (a) perceptions that the individual is a good person and (b) social distancing. Results from 5000 Bootstrap samples (bias corrected) indicated that the direct effect of response condition on perception that the individual is a good person was not significant, $B=$ $0.17, S E=0.10, p=.091,95 \%$ CI $[-0.03,0.37]$. The confidence interval for the indirect effect did not contain 0 , indicating a significant indirect effect through perceptions that the individual is prejudiced, $B=-0.31, S E=0.05,95 \% \mathrm{CI}[-0.41,-0.21]$. The direct effect of response condition 
on desire to socially distance from the individual was not significant, $B=-0.10, S E=0.09, p=$ $.238,95 \% \mathrm{CI}[-0.28,0.07]$. The confidence interval for the indirect effect did not contain 0 , indicating a significant indirect effect through perceptions that the individual is prejudiced, $B=$ $0.44, S E=0.06,95 \%$ CI $[0.32,0.56]$.

With Study 3d, we sought to investigate whether the findings from Study 3c would replicate among predominantly White participants in the context of hiring discrimination against White people. As in the previous study, we wanted to test whether participants would privilege the individual who denies having biases over the bias admitter, even when presented with evidence of discrimination toward the participant's racial ingroup. The method for Study $3 \mathrm{~d}$ was identical to that of Study 3c, except that White participants were primarily sampled $(81 \%)$ and the targets of hiring discrimination were White applicants.

In Study 3d, a $2 \times 2$ ANOVA with response condition (deny bias; admit bias but working on it, deny bias) and discrimination level (subtle, blatant) as between-subjects factors revealed main effects of response condition, $F(1,805)=124.10, p<.001, \eta p 2=.134$, and discrimination level, $F(1,805)=45.32, p<.001, \eta p 2=.053$. These main effects were qualified by an interaction between response condition and discrimination level, $F(1,805)=10.64, p=.001, \eta p 2$ $=.013$. To decompose the interaction, a series of Bonferroni corrected pairwise comparisons were performed. In the subtle discrimination conditions, the bias denier $(M=3.86, S D=1.67)$ was perceived to be significantly less prejudiced than the bias admitter $(M=5.35, S D=1.30 ; p<$ $.001,95 \% \mathrm{CI}[-1.87,-1.10])$. In the blatant discrimination conditions, the bias denier $(M=4.89$, $\left.S D_{t}=1.60\right)$ was perceived to be significantly less prejudiced than the bias admitter $(M=5.71$, $S D=1.26 ; p<.001,95 \%$ CI $[-1.20,-0.43])$ 
Next, using the same approach as previous studies, we tested whether perceptions that the individual is biased against White people mediated participants' (a) perceptions that the individual is a good person and (b) social distancing, and whether this was moderated by discrimination level. Results from 5000 Bootstrap samples (bias corrected) indicated that the direct effect of response condition on perception that the individual is a good person was significant, $B=0.22, S E=0.09, p=.012,95 \%$ CI $[0.05,0.39]$. The confidence interval for indirect effects in both the blatant $(B=-0.20, S E=0.05,95 \%$ CI $[-0.30,-0.12])$ and the subtle $(B=-0.37, S E=0.06,95 \%$ CI $[-0.49,-0.25])$ discrimination conditions did not contain 0 , indicating significant indirect effects through perceptions that the individual is prejudiced. The indirect effect was significantly stronger in the subtle discrimination condition compared to the blatant discrimination condition $\left(B_{\text {diff }}=-0.16, S E=0.06,95 \% \mathrm{CI}[-0.28,-0.06]\right)$. The direct effect of response condition on desire to socially distance themselves from the individual was also significant, $B=-0.23, S E=0.09, p=.008,95 \%$ CI $[-0.40,-0.06]$. The confidence interval for indirect effects in both the blatant $(B=0.30, S E=0.06,95 \% \mathrm{CI}[0.19,0.43])$ and the subtle $(B=0.54, S E=0.07,95 \%$ CI $[0.40,0.70])$ discrimination conditions did not contain 0, indicating significant indirect effects through perceptions that the individual is prejudiced. The indirect effect was significantly stronger in the subtle discrimination condition compared to the blatant discrimination condition $\left(B_{\text {diff }}=0.24, S E=0.08,95 \%\right.$ CI $\left.[0.09,0.40]\right)$. 


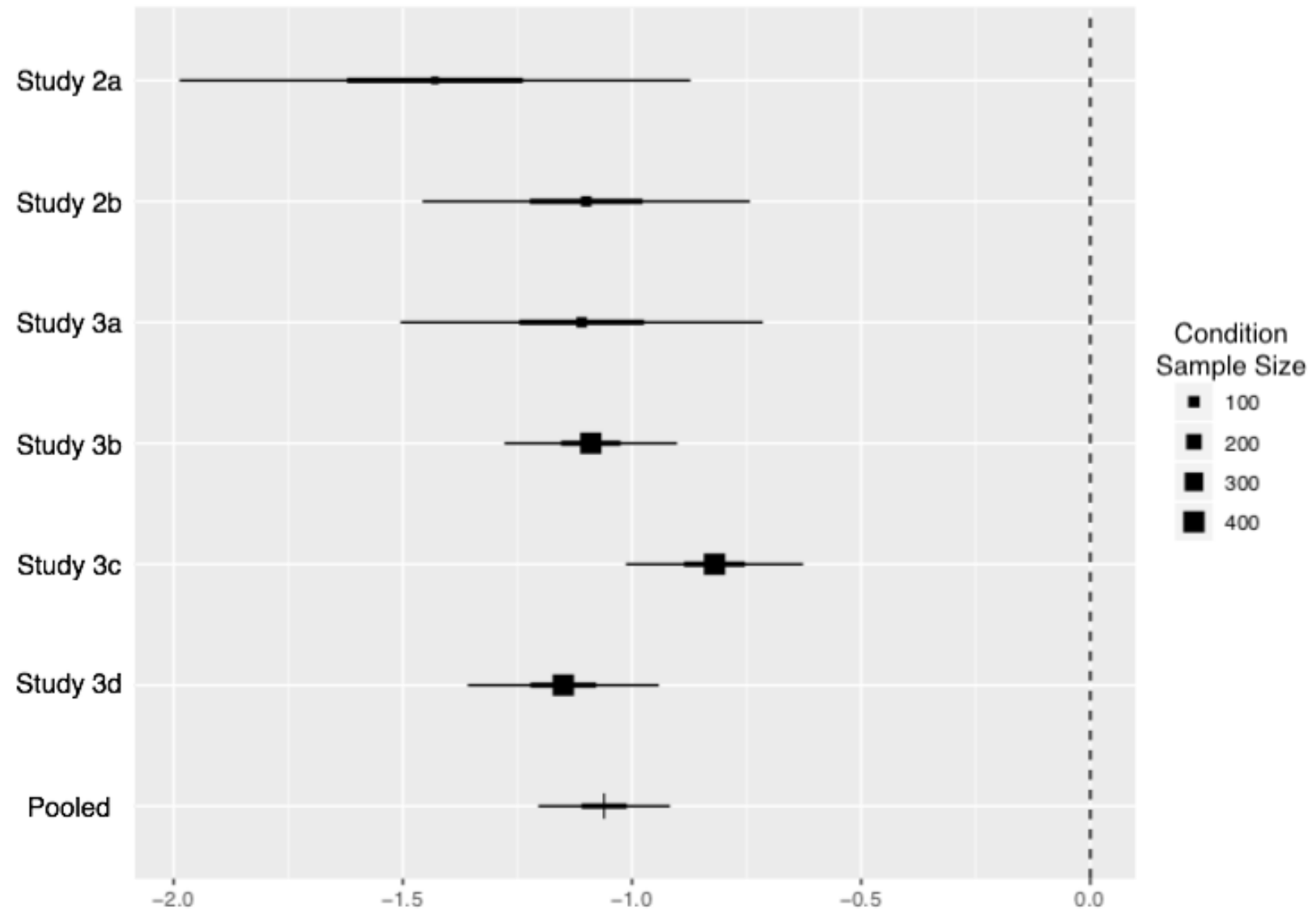

Fig. 2. The effect of denying (vs. admitting) bias on perceptions of prejudice. The plot represents the contrast estimates (unstandardized) and 95\% confidence intervals between perceiving the individual who denied (vs. admitted) having bias as being prejudiced for Studies 2a-3d. Negative values indicate lower average ratings of prejudice of the individual who denied having bias relative to individual who admitted having bias but was working on it.

Across eight studies, we found evidence that, although people tend to favor those who admit their biases when making relative comparisons, when evaluating individuals in isolation they see someone who denies having biases as less prejudiced and generally tend to favor them by viewing them as a better person and desiring less social distance from them. We observed this pattern, in spite of the information provided, indicating that both deniers and admitters do in fact 
have biases. This pattern of results emerged for both race and gender biases. Moreover, we found this same pattern across multiple instantiations of bias-deniers who demonstrated having bias on indirect measures and those who showed both blatant and subtle behavioral evidence of bias (i.e., hiring discrimination) were given the benefit of the doubt when evaluated in isolation, and these outcomes occurred when the target of discrimination was either an ingroup member or an outgroup member.

Consistent with our hypothesis that perceived prejudice is the driver of people's negative perceptions of the admitter (compared to the denier), Studies 3a-3d revealed evidence of a statistical suppression effect (also known as inconsistent mediation models; Davis \& Weber, 1985; MacKinnon, Krull, \& Lockwood, 2000). In a typical mediation model, the strength of the relation between the predictor variable and the outcome variable of interest is reduced when the mediator is included in the model, revealing a smaller (and sometimes non-significant) direct effect of the predictor on the outcome and a significant indirect effect. However, when a suppression effect occurs, the direct and indirect effects of the predictor on the outcome have opposite signs (Cliff \& Earleywine, 1994; Tzelgov \& Henik, 1991), and the direct effect of the predictor on the outcome is often strengthened. Within our studies, tests for an indirect effect revealed that, because individuals who admitted their bias were perceived to be more prejudiced than those who denied their bias, they were in turn perceived to be less good and more worthy of social distancing. Yet, when prejudice was statistically accounted for in the model, the relation between admitting prejudice and these outcomes changed, such that the admitter was actually perceived more positively than the denier. These results are consistent with the findings in Studies $1 \mathrm{a}$ and $1 \mathrm{~b}$, which revealed that, when people made relative comparisons between the admitter and denier, the admitter was perceived more positively than the denier. Taken together, 
these findings suggest that the explanation for why evaluations differ when making relative comparisons versus evaluations of isolated incidents is varying perceptions of how prejudiced the individuals actually are.

The findings that deniers are favored over admitters when evaluated separately suggests that participants may be taking evidence of bias at face value in this context, unless the individual who engaged in bias challenges it. Thus, to the extent that incidents of bias are evaluated in isolation, deniers will continue to be perceived more positively than individuals who do not deny their bias. This is particularly interesting, given that this also applies to members of the group that was discriminated against. One might think that denying bias would only improve evaluations among individuals who are not a member of the group targeted by bias. Yet, our findings show that, in spite of evidence of racial discrimination, Black participants favored individuals who denied having biases against Black people, and White participants favored individuals who denied having biases against White people. Moreover, even when faced with evidence of sexism, bias denial was found to be as effective at convincing women that the denier was lower in bias as it was with men.

The ambivalence of reactions to deniers and admitters was captured by the context in which these individuals were compared. Given that subjective perceptions of people depend upon the comparison group (Biernat \& Manis, 1994; Biernat, 2012; Kahneman \& Miller, 1986), we suggest it is plausible that observers may shift their subjective perceptions and evaluations of people when reviewing a single individual in isolation as compared to when making relative judgments of two individuals, together. As such, when making a relative comparison to an admitter of social bias (Study 1), the denier was perceived more negatively, perhaps for not being willing or able to admit their fault. In this case, the evidence of prejudice may have been 
held constant or considered undeniable. However, when making isolated social judgments with no relative comparison provided (Studies 2 and 3), the standard of what was considered prejudiced may have shifted to benefit the ostensible insight of the denier. That is, when people are asked to evaluate either a person who denies bias or a person who admits bias in isolation, people may be prone to believe what the person discloses, and thus evaluate the admitter more negatively than the denier. Indeed, we found ancillary evidence suggestive of this explanation. As reported in the supplemental analyses, participants perceived the evidence of bias presented (e.g., the hiring discrimination scenario) as a significantly less valid indicator of prejudice when reviewing the denier in isolation compared to the admitter. Although when reviewing the denier and admitter together, the majority of participants viewed both the subtle (58\%) and blatant (70\%) evidence of bias as valid indicators of prejudice. Determining whether standards of what is considered prejudice shifts depending upon the relative comparison of targets awaits further study.

Our findings potentially have broader theoretical implications regarding how people interpret and evaluate disclosure of other people's self-relevant information. Specifically, these results indicate that - because there is no way to directly access information about other's internal thoughts and feelings_- people may be inclined to believe others' self-reports over contrary evidence. The tendency to privilege self-reports may be reasoned because individuals are particularly accurate in assessing their own internal traits due to having private access to information about their own thoughts and feelings (Vazire, 2010). Thus, when there is little other information to go off of (including a relative comparison to someone who responds differently), people may intuitively believe they are using the person's self-reported insight to temper their perceptions of them. What may be missing from perceivers' calculations is how social norms and 
the social desirability to appear non-prejudiced may be influencing the person's ability and motivation to accurately report self-knowledge. However, our findings also indicate that, when people are evaluated at the same time, others may be less willing to give people who deny undesirable characteristics and behavior the benefit of the doubt. The context in which judgments are being made with a relative comparison may serve as an additional source of information that suggests the denier is not accurately reporting on his or herself.

Given that our results suggest that, at least in the context in which people are evaluated in isolation, explicitly acknowledging that one has biases may have several unintended negative social consequences, one might wonder why they shouldn't just deny their biases. Evidence suggests that, among dominant groups (e.g., White individuals), a willingness to both personally acknowledge and confront their prejudiced tendencies (as opposed to denying and/or justifying them) may lead to the self-regulation of biased attitudes and responses (Monteith et al., 2010) and reduced stereotyping (Moskowitz \& Li, 2011). Moreover, increased willingness to acknowledge (and express concern about) one's biases is associated with perceptions that subtle biased behavior can be a form of prejudice (Perry, Murphy, \& Dovidio, 2015), and an increased willingness to talk about (racial) bias to others (Perry, Skinner, \& Abaied, 2019). Thus, acknowledging and expressing concerns about one's biased tendencies may not only result in one engaging in less biased behavior, but may also increase people's exposure to others talking about their biases, and prejudice, in general. That is, if acknowledgment of the existence of and the problem with having social biases becomes more normative, people may be less likely to negatively judge those who admit their bias, and potentially more willing to admit their own biases. This is important because our results suggest that, until admitting one's biases becomes more normative, awareness of the social penalties may actually prevent people from recognizing 
and acknowledging their biases. Lastly, denial of social bias, more broadly, perpetuates social inequality wherein oppression curiously persists without the oppressors (e.g., see Kendi, 2019). Another possible reason people might be inclined to accept self-reports of others is that challenging them may require that they reconsider their own potential biases. This explanation is consistent with the aversive experience egalitarian individuals have when confronting the idea that they may hold biases (Dovidio \& Gaertner, 2000); which motivates them to distance themselves from circumstances that would clearly implicate them as biased. Thus, people may be motivated to raise the standard of what is considered to be biased to ensure that they cannot be personally deemed as biased. Although this is not a likely explanation for participants' observed motivation to avoid someone who admits having biases against the participants' group, they may similarly be inclined to believe their self-reports. Instead, participants who are evaluating someone with potential biases against their group (e.g., Black individuals evaluating discrimination against Black individuals, Study 3c) may be motivated to avoid people who explicitly acknowledge having biases against their group. For example, Black individuals might be avoidant of people who admit their biases against Blacks because they fear that the interaction be less comfortable (e.g., the person might bring up their personal biases) relative to someone who denies their biases. Future interracial interactions studies could explore this question.

Because the race and gender of the individual denying or admitting bias was not specified, it is possible that participants could have assumed that these were intergroup biases (e.g., the person showing gender bias was male and the person showing anti-Black bias was White), or intragroup biases (e.g., the person showing bias was a member of the group toward which they held bias). People's perceptions might differ depending upon the race and gender of 
the individual admitting biases. Future research should test whether target and perceiver-level characteristics (and their interaction) matter in how admitters and deniers of bias are evaluated.

The current findings provide robust support for the idea that, in the real world wherein disclosures of biases are typically made in an isolated fashion - if the goal is to minimize perceptions of social bias and protect one's reputation-bias denial might work. In spite of social psychological evidence that admitting and confronting the existence of one's biases is a step toward reducing those biases — we provide evidence that lay-people do not always see it that way. One might expect that people would generally see a person who fesses up to and expresses concerns about their wrongdoing to be the "bigger" person, and thus evaluate them more positively. Yet, we found that people only perceived the admitter more positively when they were evaluating the admitter and denier at the same time. When making judgments of those who denied and admitted social bias separately, people viewed the remorseful admitter to be more prejudiced and less favorable than the unapologetic denier.

\section{Method}

\section{Study 1}

Participants. Participants were recruited from Amazon's Mechanical Turk (MTurk) in exchange for $\$ 0.50$. The Study 1a sample was made up of 399 participants $(51.1 \%$ women $)$, who racially identified as White (78.2\%), Black/African American (7.8\%), or Asian/Asian American (5.3\%). A total of $6.5 \%$ of the Study 1a sample identified as Hispanic/Latino. Participants indicated being born during the 1960s or before (17\%), 1970s (18\%), 1980s (34\%), 1990s or after $(18 \%)$, or birth decade was unreported (14\%). Participant age was not directly measured in Studies $1 \mathrm{ab}$ and $3 \mathrm{bcd}$; thus, we relied on participants' decade of birth statistics collected independently through TurkPrime to report age for these studies. The Study $1 \mathrm{~b}$ sample was made 
up of 401 participants (56.4\% women), who racially identified as White (73.4\%), Black/African American (8.9\%), or Asian/Asian American (8.9\%). A total of $6.8 \%$ of the Study $1 \mathrm{~b}$ sample identified as Hispanic/Latino. Participants indicated being born during the 1960s or before (21\%), 1970s (18\%), 1980s (28\%), 1990s or after (17\%), or birth decade was unreported (16\%). A sensitivity power analysis (two-tailed) in $\mathrm{G}^{*}$ Power indicated that a sample of 399 would provide $80 \%$ power to detect an odds ratio of 1.22 or greater.

Materials and procedure. Participants were informed that the study was an investigation of how workers make judgments about job applicants and how other Mechanical Turk workers perceive those judgments. In Study 1a, participants were told they would have the opportunity to share their opinions and thoughts about workers' judgments about the applicants. Then participants either learned that other workers engaged in subtle or blatant discrimination in hiring recommendations. In the subtle condition, the workers selected White applicants $80 \%$ of the time over equally qualified Black applicants. In the blatant condition, workers selected White applicants $100 \%$ of the time over more qualified Black applicants. Then, participants were told of two responses workers gave when given information that they had engaged in subtle or blatant hiring discrimination. In one of the responses, the worker admits that they may hold biases and it is something they need to work on. In the other response, the worker denies having biases and it is not something they need to work on. Participants were then asked whether the admitter or denier was more prejudiced, a better person, and who they would most want to distance themselves from. For each of these outcomes, participants could select one of three relative responses: the admitter was more [prejudiced] than the denier, both the admitter and denier were equally [prejudiced], or the denier was more [prejudiced] than the admitter. The procedure for Study $1 \mathrm{~b}$ was the same as Study 1a with the exception that participants were asked to make 
decisions based on how they thought others would react to the disclosure scenarios. Data and materials for all studies can be found on Open Science Framework (OSF): https://osf.io/zjxmf/?view_only=3007f1b4a8be4c46a4ae7e34a339febd.

\section{Study 2a}

Participants. Participants were recruited from Amazon's Mechanical Turk (MTurk) in exchange for $\$ 0.50$. The sample was made up of 160 participants $\left(52.5 \%\right.$ women; $M_{\mathrm{age}}=32.31$, $\left.S D_{\text {age }}=11.74\right)$, who racially identified as White $(80.6 \%)$, Black/African American $(8.1 \%)$, or Asian/Asian American (10.6\%). A total of 5.6\% of the sample identified as Hispanic/Latino. We set our sample size based on convention $(N=40$ per cell), and a sensitivity power analysis indicated that we had $80 \%$ power to detect an effect size of $\eta 2=.063$ for our primary analyses.

Materials and procedure. Participants were informed that the study was an investigation of whether people who are given personal information about themselves will discuss that information with others and how people perceive personal information about others. They were told that they would be randomly assigned to either learn information about themselves or another Mechanical Turk Worker, and that if they learned information about themselves they would have an opportunity to comment on that information, whereas if they learned information about someone else they would be asked questions about their perceptions of that person. Participants were subsequently told that they had been randomly assigned to learn information about someone else who scored (a) relatively low in self-esteem and (b) as having "a strong automatic preference for White people over Black people.” Participants were given information about the individual's self-esteem in order to reduce suspicion that our study was solely focused on bias. 
Response conditions. Participants were randomly assigned to view one of four responses that the individual ostensibly had to that information. In the admit bias condition, the individual indicated that they thought the test was accurate in picking up their racial biases and that it is something that they need to work on. In the deny bias condition, the individual rejected the results of the test, stating that they do not believe that the test is accurate and that this is not something that they need to work on. Additionally, we included two control conditions: one in which the individual did not share any information and one in which the person admitted having low self-esteem.

Outcome measures. Next, participants were asked to answer a series of questions about their attitudes toward the person they had just been given information about on 7-point scales anchored by Strongly Disagree and Strongly Agree. Items included (a) “... is racially prejudiced or biased," and (b) “...is probably a good person, overall; ” and (c) five social distancing items (with items scored such that higher scores equaled greater social distancing) including: "I would be willing to spend time with in the future" (reverse-scored), "I would not want other people to associate me with ," "I would like to have as a neighbor" (reversescored), "It would bother me to have to work with ," and "I could see myself being friends with " (reverse-scored; $M=3.66, S D=1.14, \alpha=.93$ ). Lastly, participants were given the opportunity to write any additional thoughts that they would like to share about the person. Individual difference measures (e.g., Bias Awareness, malleability of bias beliefs) were also included but not analyzed as we subsequently recognized that we had insufficient power to test these associations. 


\section{Study 2b}

Participants. Participants were recruited from MTurk in exchange for $\$ 0.50$. The sample was made up of 324 participants $\left(47.8 \%\right.$ women; $\left.M_{\text {age }}=34.95, S D_{\text {age }}=12.34\right)$, who racially identified as White (75.3\%), Black/African American (8.0\%), or Asian/Asian American (6.5\%). A total of $7.7 \%$ of the sample identified as Hispanic/Latino. We set our sample size based on convention ( $N \sim 40$ per cell). Although we still had four conditions we wanted to be able to test for effects of (gender) group membership, thus we doubled our total sample size. A sensitivity power analysis indicated that we had $80 \%$ power to detect an effect size of $\eta 2=.031$ for our primary analyses.

Materials and procedure. The cover story and opening procedures for Study $2 \mathrm{~b}$ were identical to Study 2a, except that participants were told that the person scored as having a "strong automatic preference for men over women in positions of leadership.” Participants were informed that the individual had (a) indicated that they thought the test was accurate in picking up their gender biases with regard to leadership and that it is something that they need to work on, (b) indicated that they thought the test was accurate in picking up their gender biases with regard to leadership (c) stated that they do not believe that the test is accurate and that this is not something that they need to work on, or (d) admitted having low self-esteem. Participants were asked to answer the same series of questions used in Study 2a (social distance scale, $M=3.50$, $S D=1.09, \alpha=.87)$.

\section{Study 3a}

Participants. Participants were recruited from MTurk in exchange for $\$ 0.50$. The sample consisted of 243 participants $\left(49.3 \%\right.$ women; $\left.M_{\text {age }}=34.88, S D_{\text {age }}=12.86\right)$, who racially identified as White (78.6\%), Black/African American (9.9\%), or Asian/Asian American (9.5\%). 
A total of $5.3 \%$ of the sample identified as Hispanic/Latino. We set our sample size based on convention $(N \sim 40$ per cell). A sensitivity power analysis indicated that we had $80 \%$ power to detect an effect size of $\eta 2=.039$ for our primary analyses.

Materials and procedure. Participants were informed that they had been assigned to read personal information about someone who had previously read over 20 applications for a leadership position in an organization and selected 10 of those applicants. Participants learned that the pool had had an equal number of men and women and that the applicants were equally qualified and that the person had selected eight men and only two women for leadership positions, suggesting that they are biased and have a preference for men over women in positions of leadership. Then participants were randomly assigned to view one of three responses to that information (deny bias, admit bias and working on it, or only admit bias). The outcome measures in this study were identical to those in the previous studies (social distance scale, $M=3.60, S D=$ $1.09, \alpha=.88)$.

\section{Study 3b}

Participants. Participants were recruited from MTurk in exchange for $\$ 1.50$. We sought to recruit approximately 788 participants for this $2 \times 2$ between-subjects design, with approximately 197 participants in each condition. This sample size was based on a power analysis conducted using $\mathrm{g}^{*}$ power, indicating that in order to detect a small effect $(\eta 2=.01)$ with $80 \%$ power and an alpha of .05 we needed to recruit this many participants. The final sample consisted of 807 participants (52\% women), who racially identified as White $(72 \%)$, Black/African American (10\%), Asian/Asian American (7\%), or Other (2\%). A total of 8\% of the sample identified as Hispanic/Latino. Participants indicated being born during the 1960s or 
before $(14 \%), 1970$ s $(16 \%), 1980$ s $(33 \%), 1990$ s or after $(20 \%)$, or birth decade was unreported $(18 \%)$.

Materials and procedure. Study $3 \mathrm{~b}$ was identical to Study $3 \mathrm{a}$ with the exception of the following changes. With Study $3 \mathrm{~b}$ we sought to investigate whether the findings from Study $3 a$ would replicate when a Black relative to White applicant was discriminated against. However given that the admit working on it and admit (no mention of working on it) conditions did not tend to differ, we dropped the admit (no mention of working on it) condition from Study $3 b$. Finally, we added a blatant bias condition to Study 3 b, to assess whether the effects would replicate when the individual engaged in a more blatant form of discrimination. The method for Study $3 b$ was identical to that of Study $3 a$, except participants in the blatant bias condition were assigned to evaluate a scenario in which another MTurk worker ostensibly recommended hiring ten White candidates and zero Black candidates for a leadership job (out of ten White and ten Black candidates). Importantly, participants were told that the Black candidates were objectively more qualified for the position than the White candidates, although the individual who evaluated them was never informed of that. As with the previous study, participants in the subtle bias condition were assigned to evaluate a scenario in which another MTurk worker ostensibly selected eight White and two Black candidates for a leadership job (out of ten White and ten Black, equally qualified candidates). The methods and analysis plan were preregistered on Open Science Framework prior to data collection: https://osf.io/ryqsk/?view_only=b5084571b09c49abbf07ecdf81af23fa.

\section{Study 3c}

The sample size was determined using the same criteria as Study 3b. Participants were recruited from MTurk in exchange for $\$ 1.50$. The sample consisted of 796 participants $(69.1 \%$ 
women), who racially identified as Black/African American (98.7\%) and non-Black (1.3\%). A total of $1.4 \%$ of the sample identified as Hispanic/Latino. Although we requested TurkPrime to only allow for Black/African American workers to qualify for this study, demographic responses on the Qualtrics survey indicated that not quite all of the sample self-identified as Black/African American (98.7\%). Participants indicated being born during the $1960 \mathrm{~s}$ or before $(13 \%)$, 1970s $(18 \%), 1980$ s (31\%), 1990s or after (21\%), or birth decade was unreported (17\%). With Study 3c, we sought to investigate how Black participants would evaluate someone who admitted compared to denied their bias using a predominantly Black sample. Specifically, we were interested in assessing whether Black participants would also evaluate those who admitted their anti-Black biases more negatively than those who denied them. Our hypotheses and methods for this study were preregistered with the Open Science Framework:

https://osf.io/a26qn/?view_only=07cd79adf5ec4cabbb0f0a0da83056ec. The method for Study 3c was identical to that of Study 3b, except in this study we recruited participants who selfidentified Black or African American.

\section{Study 3d}

The sample size was determined using the same criteria as Study 3b. Participants were recruited from MTurk in exchange for $\$ 1.50$. The sample consisted of 803 participants $(54.7 \%$ women), who racially identified as White $(81 \%)$ and as non-White $(19 \%)$. A total of $7.2 \%$ of the sample identified as Hispanic/Latino. Participants indicated being born during the 1960s or before $(14 \%), 1970 \mathrm{~s}(16 \%), 1980 \mathrm{~s}(33 \%), 1990$ s or after $(25 \%)$, or birth decade was unreported $(12 \%)$. Although we requested TurkPrime to only allow for White workers to qualify for this study, demographic responses on the Qualtrics survey indicated that only $81 \%$ of the sample self-identified as White. The cause of this discrepancy is unclear; however, to be sufficiently 
powered, non-White participants were not excluded from pre-registered analyses. Study 3d was identical to Study 3c, with the exception of the fact that most participants identified as White and the targets of bias were White job candidates (rather than Black job candidates). 


\section{References}

Augoustinos, M., \& Every, D. (2010). Accusations and denials of racism: Managing moral accountability in public discourse. Discourse \& Society, 21, 251-256. doi:10.1177/0957926509360650

Bem, D. J. (1972). Self-perception theory. In Advances in experimental social psychology (Vol. 6, pp. 1-62). Academic Press. https://doi.org/10.1016/S0065-2601(08)60024-6

Biernat, M. (2012). Stereotypes and shifting standards: Forming, communicating, and translating person impressions. In Advances in Experimental Social Psychology (Vol. 45, pp. 1-59). Academic Press. https://doi.org/10.1016/B978-0-12-394286-9.00001-9

Biernat, M., \& Manis, M. (1994). Shifting standards and stereotype-based judgments. Journal of Personality and Social Psychology, 66, 5-20. http://dx.doi.org/10.1037/0022-3514.66.1.5

Blaut, J. M. (1992). The theory of cultural racism. Antipode, 24, 289-299. doi:10.1111/j.14678330.1992.tb00448.x

Bonilla-Silva, E. (2002). The linguistics of color blind racism: How to talk nasty about Blacks without sounding "racist." Critical Sociology, 28, 41-64. https://doi.org/10.1177/08969205020280010501

Bruk, A., Scholl, S. G., \& Bless, H. (2018). Beautiful mess effect: Self-other differences in evaluation of showing vulnerability. Journal of Personality and Social Psychology, 115, 192-205. http://dx.doi.org/10.1037/pspa0000120

Cliff N., \& Earleywine M. (1994). All predictors are "mediators" unless the other predictor is a “suppressor.” Unpublished manuscript.

Davis, J. A., \& Weber, R. P. (1985). The logic of causal order (Vol. 55). Beverly Hills, CA: Sage Publications. 
Dovidio, J. F., \& Gaertner, S. L. (2000). Aversive racism and selection decisions: 1989 and 1999. Psychological Science, 11, 315-319. https://doi.org/10.1111/1467-9280.00262

Gilbert, D. T., \& Malone, P.S. (1995). The correspondence bias. Psychological Bulletin, 117, 21-38. http://dx.doi.org/10.1037/0033-2909.117.1.21

Grube, J. W., Mayton, D. M., \& Ball-Rokeach, S. J. (1994). Inducing change in values, attitudes, and behaviors: Belief system theory and the method of value self-confrontation. Journal of Social Issues, 50, 153-173. https://doi.org/10.1111/j.1540-4560.1994.tb01202.x

Hayes, A. F. (2017). Introduction to Mediation, Moderation, and Conditional Process Analysis: A Regression-Based Approach. Guilford Publications.

John, L. K., Barasz, K., \& Norton, M. I. (2016). Hiding personal information reveals the worst. Proceedings of the National Academy of Sciences, 113, 954-959. https://doi.org/10.1073/pnas.1516868113

Kahneman, D., \& Miller, D. T. (1986). Norm theory: Comparing reality to its alternatives. Psychological review, 93, 136-153. https://doi.org/10.1037/0033-295X.93.2.136

Kendi, I. X. (2019). How to Be an Antiracist. New York: One World.

Kim, P. H., Ferrin, D. L., Cooper, C. D., \& Dirks, K. T. (2004). Removing the shadow of suspicion: The effects of apology versus denial for repairing competence- versus integrity-based trust violations. Journal of Applied Psychology, 89, 104-118. http://dx.doi.org/10.1037/0021-9010.89.1.104

MacKinnon, D. P., Krull, J. L., \& Lockwood, C. M. (2000). Equivalence of the mediation, confounding and suppression effect. Prevention Science, 1, 173-181. doi: 10.1023/A:1026595011371 
Monteith, M. J., Ashburn-Nardo, L., Voils, C. I., \& Czopp, A. M. (2002). Putting the brakes on prejudice: On the development and operation of cues for control. Journal of Personality and Social Psychology, 83, 1029. doi:10.1037//0022-3514.83.5.1029

Monteith, M. J., \& Mark, A. Y. (2009). The self-regulation of prejudice. Handbook of Prejudice, Stereotyping, and Discrimination, 507-523.

Monteith, M. J., Mark, A. Y., \& Ashburn-Nardo, L. (2010). The self-regulation of prejudice: Toward understanding its lived character. Group Processes \& Intergroup Relations, 13, 183-200. https://doi.org/10.1177/1368430209353633

Monteith, M. J. (1993). Self-regulation of prejudiced responses: Implications for progress in prejudice-reduction efforts. Journal of Personality and Social Psychology, 65, 469-485. http://dx.doi.org.turing.library.northwestern.edu/10.1037/0022-3514.65.3.469

Moskowitz, G. B., \& Li, P. (2011). Egalitarian goals trigger stereotype inhibition: A proactive form of stereotype control. Journal of Experimental Social Psychology, 47, 103-116. https://doi.org/10.1016/j.jesp.2010.08.014

Moskowitz, G. B., Li, P., Ignarri, C., \& Stone, J. (2011). Compensatory cognition associated with egalitarian goals. Journal of Experimental Social Psychology, 47, 365-370. https://doi.org/10.1016/j.jesp.2010.08.010

Noon, M. (2018). Pointless diversity training: unconscious bias, new racism and agency. Work, Employment and Society, 32, 198-209. https://doi.org/10.1177/0950017017719841

Penner, L.A. (1971). Interpersonal attraction toward a black person as a function of value importance. Personality: An International Journal, 2, 175-187.

Perry, S. P., Murphy, M. C., \& Dovidio, J. F. (2015). Modern prejudice: Subtle, but unconscious? The role of bias awareness in Whites' perceptions of personal and others' 
biases. Journal of Experimental Social Psychology, 61, 64-78. http://doi.org/10.1016/j.jesp.2015.06.007

Perry, S., Skinner, A., \& Abaied, J. (2019). Bias awareness predicts color conscious racial socialization methods among White parents. Advance online publication. Journal of Social Issues. https://doi.org/10.1111/josi.12348

Redford, L., \& Ratliff, K. A. (2016). Perceived moral responsibility for attitude-based discrimination. The British Journal of Social Psychology, 55, 279-296. http://doi.org/10.1111/bjso.12123

Richeson, J. A., \& Shelton, J. N. (2003). When prejudice does not pay: Effects of interracial contact on executive function. Psychological Science, 14, 287-290. https://doi.org/10.1111/1467-9280.03437

Rokeach, M., \& Cochkane, R. (1972). Self-confrontation and confrontation with another as determinants of long-term value change. Journal of Applied Social Psychology, 2, 283292. https://doi.org/10.1111/j.1559-1816.1972.tb01280.x

Shelton, J. N. (2003). Interpersonal concerns in social encounters between majority and minority group members. Group Processes and Intergroup Relations, 6, 171-186. https://doi.org/10.1177/1368430203006002003

Spence, J. T., \& Hahn, E. D. (1997). The attitudes toward women scale and attitude change in college students. Psychology of Women Quarterly, 21, 17-34. doi:10.1111/j.14716402.1997. tb00098.x

Wheeless, L. R., \& Grotz J. (1977). The measurement of trust and its relationship to selfdisclosure. Human Communication Research, 3, 250-257. https://doi.org/10.1111/j.1468-2958.1977.tb00523.x 
Vazire, S. (2010). Who knows what about a person? The self-other knowledge asymmetry (SOKA) model. Journal of Personality and Social Psychology, 98, 281-300.

Twenge, J. M. (1997). Attitudes toward women, 1970-1995: A meta-analysis. Psychology of Women Quarterly, 21, 35-52. doi:10.1111/j.1471-6402.1997.tb00099.x

Tzelgov, J., \& Henik, A. (1991). Suppression situations in psychological research: Definitions, implications, and applications. Psychological Bulletin, 109, 524-536.

doi:10.1037/a0017908 